\section{The Nuclear Spin of Arsenic}

ThE fine structures occurring in the lines of the spectrum of As II have been observed by me in the region $\lambda \lambda 6400-4300$ by means of a Fabry.Perot interferometer with plate separations varying from $5 \mathrm{~mm}$. to $25 \mathrm{~mm}$. The source employed was a Geissler tube containing arsenic, helium at a pressure of $2 \mathrm{~mm}$. being circulated through the tube. Fifty lines have been observed, thirty-three of which are single, the rest showing fine structure. Most of the lines showing structure involve transitions between the $4 p 5 s$ and $4 p 5 p$ electron configurations and divide themselves into two distinct groups. These are, first, degraded regular series, which arise from the fact that only one of the terms involved, usually the $4 p 5 s$ term, shows appreciable structure; and secondly, complex patterns due to appreciable structure in both upper
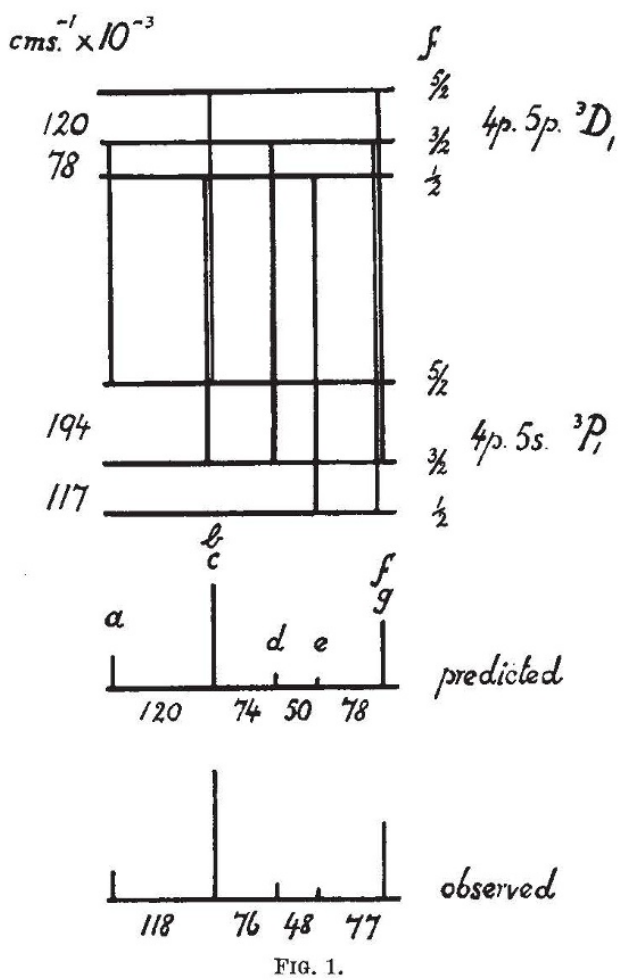

and lower terms. The gross multiplet structure has been analysed by Dr. K. R. Rao, who has kindly allowed me to use his unpublished data, and the fine structure observations confirm the gross structure analysis in detail.

Of the $4 p 5 s{ }^{3} P$ terms, the terms with $j=0$ are single, those with $j=1$ are triple, and those with $j=2$ are quadruple. The nuclear spin is therefore $\frac{: 3}{2}$. This value is amply confirmed by other data, and the fine structure analyses of the various lines check one another extremely well. For example, the line $4 p 5 s{ }^{3} P_{0}-4 p 5 p^{3} D_{1}$ is a regular triplet degraded to the red, the intervals being 120 and $78 \mathrm{~cm}^{-1} \times 10^{-3}$. This is therefore the term structure for $4 p 5 p{ }^{3} D_{1}$, since $4 p 5 s{ }^{3} P_{0}$ remains single. Also, the line $4 p 5 s{ }^{3} P_{1}-$ $4 p 5 p{ }^{3} P_{1}$ is an extremely sharp triplet degraded to the violet, with intervals 194, I17. Other data prove that the sitructure of the $4 p 5 p{ }^{3} P_{1}$ term is quite negligible, so that this gives the structure of the $4 p 5 s{ }^{3} P_{1}$ term. From these the fine structure of the line $4 p 5 s{ }^{3} P_{1}-4 p 5 s{ }^{3} D_{1}$ can be predicted, and, as shown in Fig. 1, the agreement with the structure actually observed is remarkably exact, both as to intensities and intervals. Since the components $b c$ and $f g$ respectively fall right on top of one another, their intensities have been added in forming the predicted pattern.

Attention may be directed to the relatively coarse structure of the $4 p 5 p^{3} D_{1}$ term as compared with the $4 p 5 s$ terms. Since this term possesses no penetrating $s$ electron, it is to be expected at first that the structure will be small. However, Breit ${ }^{1}$ has shown that in the heavier atoms a $p_{\frac{1}{2}}$ electron behaves with respect to the nucleus as if it were penetrating, and since the $4 p 5 p{ }^{3} D_{1}$ term is built up out of two $p_{\frac{1}{2}}$ electrons with parallel coupling, this may be the correct explanation of the relatively coarse structure, as the atomic weight of arsenic is 79. A detailed account of the fine structures will be published elsewhere shortly.

Physikalisch-Technische Reichsanstalt, S. TOLANSKY.

\section{Berlin-Charlottenburg,} March 14.

1 Breit, Phys. Rev.. 37, 1182; 1931.

\section{Infra-Red Absorption Spectra of the Oxides of Chlorine}

We have recently completed an examination of the infra-red absorption spectra of the monoxide and dioxide of chlorine. The unstable nature of these substances demanded the use of a monochromator method: this has the additional advantage of an increased purity of spectrum, but even under these conditions there is a considerable amount of scattered radiation in the region of longer wave-lengths. We have endeavoured to take advantage of the alteration in the chromatic foci of rocksalt to stop out most of the scattered light, but owing to the lack of transparency of this substance beyond $18 \mu$ (the optical train included rocksalt lenses and plates for the absorption cells and thermopile case), we obtained no measurable deflections on the galvanometer. The provision of optical parts of potassium bromide should eventually solve this difficulty. The observed bands for $\mathrm{ClO}_{2}$ are recorded in the accompanying Table.

\begin{tabular}{|c|c|c|c|c|c|}
\hline Band. & $(\lambda) \mu$. & $\begin{array}{c}\text { Maxima } \\
\left(\mathrm{cm} .^{-1}\right)\end{array}$ & $\begin{array}{c}P-R \\
\Delta \nu\left(\mathrm{cm} .^{-1}\right)\end{array}$ & $\begin{array}{c}\text { Relative } \\
\text { Intensity. }\end{array}$ & Origin. \\
\hline$B$ & 10.57 & $\begin{array}{r}932 \\
963\end{array}$ & 31 & 30 & $\nu_{2}$ \\
\hline & & $\begin{array}{r}1095 \\
1105\end{array}$ & 28 & 80 & $\nu_{3}$ \\
& 9.017 & $\begin{array}{r}1123 \\
1870\end{array}$ & & & \\
$D$ & 5.307 & $\begin{array}{l}1884 \\
1900\end{array}$ & 30 & 0.5 & $2 \nu_{2}$ \\
$E$ & 4.916 & 2034 & - & 4 & $\nu_{2}+\nu_{3}$ \\
$F$ & 4.246 & 2355 & - & 0.5 & $2 \nu_{3}$ \\
\hline
\end{tabular}

$v_{1}$ for this molecule falls outside the range of our spectrometer ; recent work by Urey and Johnston ${ }^{1}$ gives a probable value of $529 \mathrm{~cm}^{-1}$ for this fundamental. The spectrum is very similar indeed to that of sulphur dioxide; this fact is perhaps to be connected with the 'odd ' electron structure of $\mathrm{ClO}_{2}$, there being no corresponding proper function in this case to satisfy the odd electron, and except for the instability associated with this, we have for the rest of the molecule much the same external electronic structure in the two substances.

We have extended the examination of the possible modes of vibration for the symmetrical triangular

$$
\text { No. 3261, VoL. 129] }
$$

